\title{
Percepciones de Docentes y Padres sobre la Educación Inclusiva y las Barreras para su Implementación en Lima, Perú
}

\section{Parents' and Teachers' Perceptions of Inclusive Education and the Barriers to its Implementation in Lima, Peru}

Paola Ruiz-Bernardo *

Universitat Jaume I

\begin{abstract}
Este artículo expone los resultados de una investigación llevada a cabo en Lima, Perú, con el objetivo de conocer y reflexionar sobre cómo se está implementando la educación inclusiva y cuáles son sus barreras y propuestas de acción para alcanzarla. La investigación es de corte claramente cualitativo y pretende indagar y reflexionar junto a los actores educativos y sociales, vinculados a la escuela, sobre sus percepciones de la educación inclusiva y en función de estas reflexiones favorecer un empoderamiento para la mejora de sus prácticas y la transformación del centro escolar y el entorno social, desde una crítica al actual modelo de escuela excluyente y bajo la orientación de una escuela más justa e inclusiva. La recogida de información ha sido mediante: grupos de discusión, entrevistas y diario de observación de la investigadora. Para la organización y presentación de datos se ha utilizado el Atlas.ti. Los principales hallazgos se centran en que todos los participantes conciben la educación inclusiva como la incorporación de los alumnos con NEE en las escuelas ordinarias. Que las principales barreras son la falta de sensibilización social acerca de la exclusión, la falta de formación docente especialmente en estrategias y prácticas inclusivas, y la necesidad de disminuir la ratio de alumnos por docentes. Por tanto, las propuestas de mejora están en la línea de solventar los problemas detectados.
\end{abstract}

Descriptores: Educación inclusiva, Educación intercultural, Percepciones de agentes educativos, Investigación cualitativa.

This article presents the results of a research carried out in Lima, Peru. The aim of this study was to know and reflect on the implementation of inclusive education, its barriers and the proposals for action to achieve it. Research is clearly qualitative and aims to investigate and reflect, together with educational and social actors related to school, about their perceptions of inclusive education and promote empowerment to improve their practices and the transformation of the school and the social environment. Considered from a critique of the current model of exclusionary school and under the guidance of a fairer and more inclusive school. The collection of information has been done through: focus groups, interviews and observation diary of the researcher. Atlas.ti has been used for the organization and presentation of the data. The main findings focus on the conception of inclusive education as the inclusion of students with special needs in mainstream schools. The main barriers are the lack of social awareness towards exclusion, lack of teacher training especially in strategies, and the need to reduce the ratio of students per teacher. Therefore, the proposed improvements are in line to solve the problems detected.

Keywords: Inclusive education, Intercultural education, Perceptions of educational agents, Qualitative research.

Esta investigación se ha realizado con la ayuda otorgada por el Plan de Promoción de la Investigación del 2015, de la Universitat Jaume I de Castellón.

*Contacto: ruizma@uji.es

ISSN: 0718-7378

www.rinace.net/rlei/
Recibido: 27/02/2016

$1^{\text {a }}$ Evaluación: 28/06/2016

Aceptado: 23/08/2016 


\section{Introducción}

Hablar de educación inclusiva siempre implica tener que aclarar los términos, pues tal como lo ha manifestado Echeita y Sandoval (2002), Parrilla (2002) o Ainscow (2004), entre otros, no existe una definición única, sino que es un término aglutinador con diferentes acepciones de la misma. Además, hay que considerar que este término de ambigua interpretación está atravesado por las experiencias culturales y las prácticas educativas de cada lugar. Por lo menos esta ha sido la experiencia encontrada al recorrer diversos países de Europa y de Latinoamérica. En concreto en este artículo se trata de abordar cómo se entiende y, en consecuencia, se lleva a la práctica, la educación inclusiva, en las escuelas de Lima, Perú. Y también identificar las que creen (sus actores) que son las barreras y las posibles acciones para superarlas. No es un análisis exhaustivo de dicha realidad sino una aproximación para que ayude a sus actores a la reflexión y posterior acción en pro de la educación inclusiva.

En la última década han sido muchos los países europeos y latinoamericanos (Ramos, 2013; Padin, 2013) que han reformulado sus políticas educativas y en todas ellas aparece claramente la adhesión de lo que se conoce (y luego cada uno interpreta) como educación inclusiva.

Un ejemplo de esto lo vemos en el artículo 2, sobre el derecho de la educación, del reglamento de la ley n 28.044, Ley General de Educación (2012), de Perú, el cual cita explícitamente como una característica de la educación: la inclusividad y la define como la posibilidad de acceder, e indirectamente, de permanecer en el sistema educativo, la misma dice:

Inciso b) El derecho de acceso a una educación de calidad, equitativa, pertinente e inclusiva, y a una educación pública gratuita. La accesibilidad implica la inclusión en el sistema educativo de personas con necesidades educativas especiales y de grupos sociales excluidos y vulnerables, sin distinción de etnia, religión, sexo u otra causa de discriminación. Además, la institución educativa asegura la permanencia en el servicio educativo de las adolescentes embarazadas o madres y los estudiantes con VIH y adopta medidas que garanticen su inclusión sin discriminación. (p. 2)

Inciso c) El derecho a la permanencia, por el cual los estudiantes deben contar con la oportunidad y las facilidades para continuar en la institución educativa, o reingresar a ella, sin que sus condiciones personales, socioeconómicas o culturales sean un obstáculo o impedimento. (p. 2)

Este es el punto de inicio para empezar a estudiar cómo estas palabras se concretan en el aula de las instituciones educativas de Lima, Perú.

Por ello esta investigación tratará de dar respuestas a las siguientes cuestiones: ¿Cómo se entiende la educación inclusiva en Perú?, ¿en la práctica cómo se concreta en las aulas?, ¿cuáles son las barreras que se identifican para su aplicación? y ¿qué se puede hacer para mejorar la escuela de cara a la educación inclusiva? 


\section{El sistema educativo en Perú. Soles y sombras}

El Estado peruano tiene una población estimada para el 2015 de 31.151.643 habitantes (INEI, 2015a). La educación obligatoria abarca de 6 a 16 años, siendo la tasa bruta ${ }^{1}$ de escolarización para primaria de 99,2\% y de secundaria de 93,6\%.

De acuerdo con las estadísticas educativas proporcionadas por el Instituto Nacional de Estadística e Informática (INEI, 2015b), se destaca la siguiente información:

- Con respecto a la educación infantil: el acceso a la educación inicial se ha incrementado paulatinamente tal como lo muestra la figura 1 entre los años 2005 a 2014.

- Respecto al nivel primario, en el año 2014, el 92,1\% (tasa neta) de la población de 6 a 11 años de edad estuvo matriculada en el nivel de estudios que le corresponde a su edad normativa. En cambio, la tasa bruta de matrícula de este grupo de edad alcanzó el 99,2\%, es decir de cada 100 niñas y niños de 6 a 11 años de edad, 92 están matriculados en el nivel que le corresponde a su edad normativa, 7 están matriculados en algún nivel de educación y 1 no está matriculado.

- Finalmente, en el nivel secundario, durante el año 2014, la tasa neta de matrícula escolar de la población de 12 a 16 años de edad, se situó en 83,1\%, mostrando un crecimiento de 1,3 puntos porcentuales respecto al año anterior. La tasa bruta de matrícula de este grupo de edad se ubicó en $93,6 \%$.

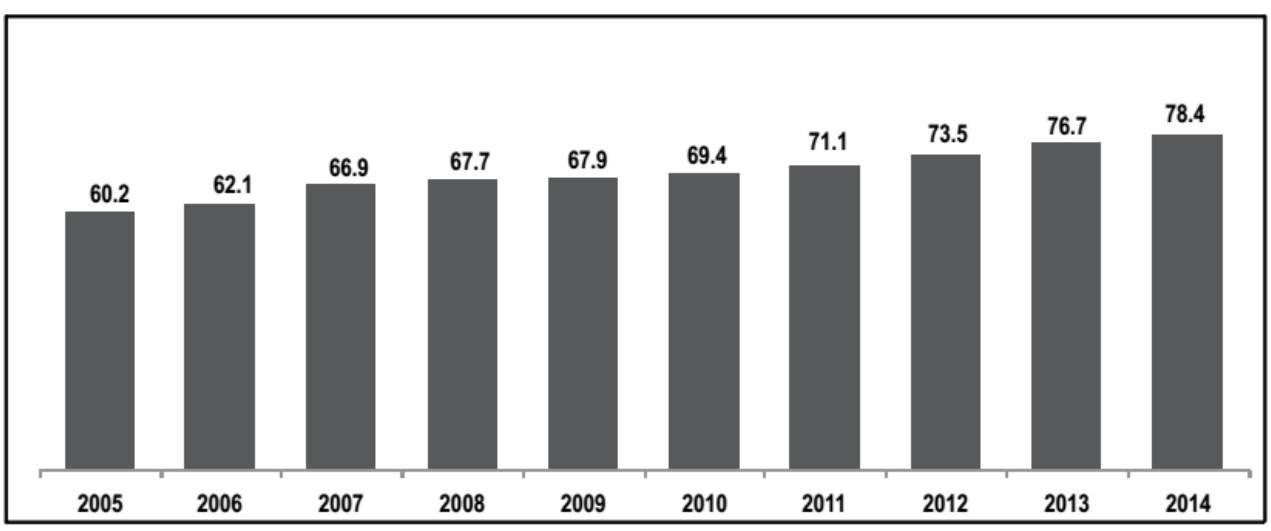

Figura 1. Perú: tasa neta de matrícula escolar de la población de 3 a 5 años. 2005-2014 Fuente: Instituto Nacional de Estadística e Informática. Encuesta nacional de hogares.

A partir de lo que se entiende con respecto a una educación para todos (Booth y Ainscow, 2015; UNESCO/MEC, 1994), se ha prestado especial atención a las estadísticas que se refieren al incremento de matrículas asociadas a las condiciones socioeconómicas durante la última década. Se puede rescatar la siguiente información relevante (figuras 2, 3 y 4$)$ :

\footnotetext{
${ }^{1}$ La tasa bruta es la matrícula de la población en edad escolar a algún nivel o grado de estudios del sistema educativo. En cambio, la tasa neta refleja la matrícula de la población en edad normativa al nivel de estudios que le corresponde.
} 
Tasa neta de matrícula escolar de la población de 3 a 5 años de edad según condición socioeconómica: Al analizar la matrícula desagregada por condición socioeconómica del hogar, se observan diferencias significativas. La mayor tasa neta de matrícula se presenta en el estrato socioeconómico más alto ( $V$ quintil) con 91,1\%, frente a 71,6\% registrado en el quintil más pobre (I quintil), con una brecha de 19,5 puntos porcentuales. Si comparamos la matrícula escolar del año 2014 con lo registrado en el 2013 se aprecia un incremento de (3,0 puntos porcentuales) tanto en el estrato más pobre, como en el estrato más rico. (INIE, 2015, p. 32)

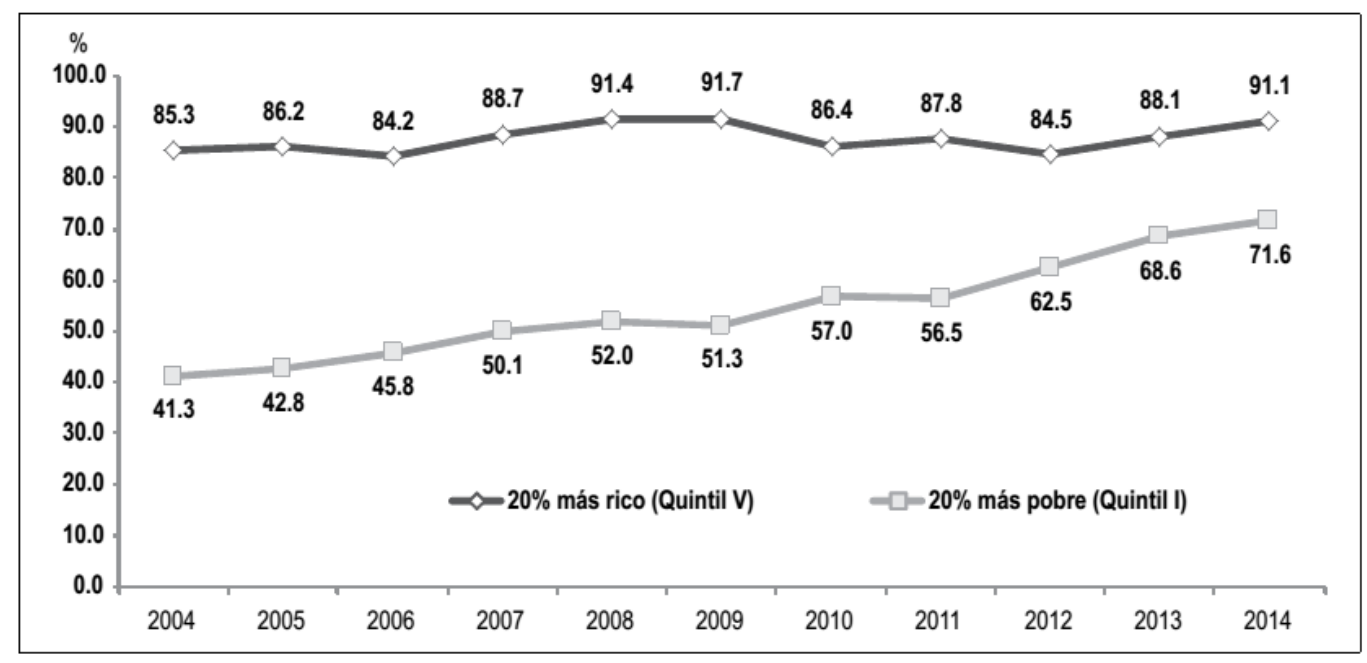

Figura 2. Perú: tasa neta de matrícula a educación inicial, según condición socioeconómica, 2004-2014

Fuente: Instituto Nacional de Estadística e Informática. Encuesta nacional de hogares.

Tasa de matrícula escolar de la población de 6 a 11 años de edad según condición socioeconómica: En todos los estratos sociales la tasa neta de matrícula a educación primaria es alta, existiendo pocas diferencias entre quintiles. Así, en el primer quintil la tasa neta llega a 92,2\% y en el quinto quintil a 92,4\%. Lo que evidencia el acceso igualitario y universal a la educación primaria. (INIE, 2015, p. 37)

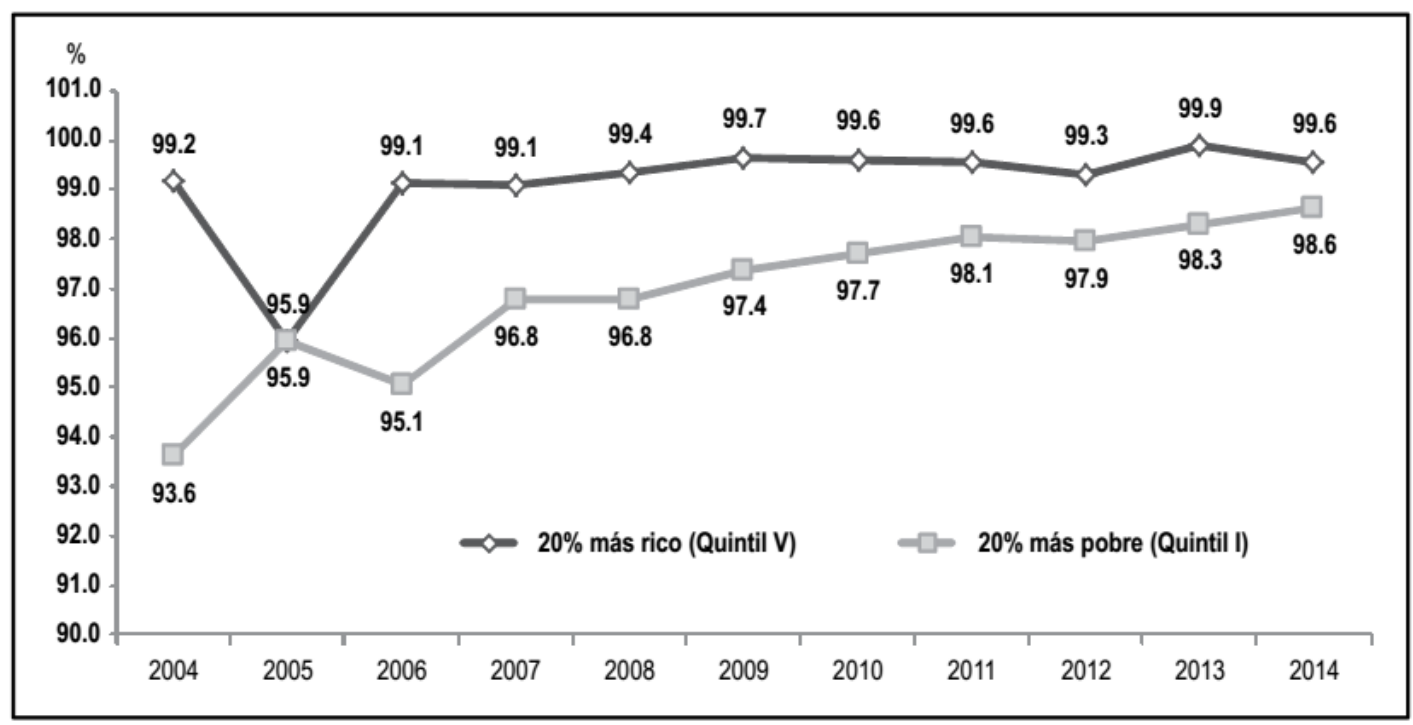

Figura 3. Perú: tasa neta de matrícula escolar de la población de 6 a 11 años según condición socioeconómica, 2004-2014

Fuente: Instituto Nacional de Estadística e Informática. Encuesta nacional de hogares. 
Tasa de matrícula escolar de la población de 12 a 16 años de edad, según condición socioeconómica: Se observa brechas importantes entre los estratos sociales. Así, la tasa neta de matrícula a educación secundaria del primer quintil (20\% más pobre) fue de 73,5\%, siendo 17,8 puntos porcentuales menos que en el quinto quintil (20\% más rico) que alcanzó el 91,3\%. (INIE, 2015, p. 42)

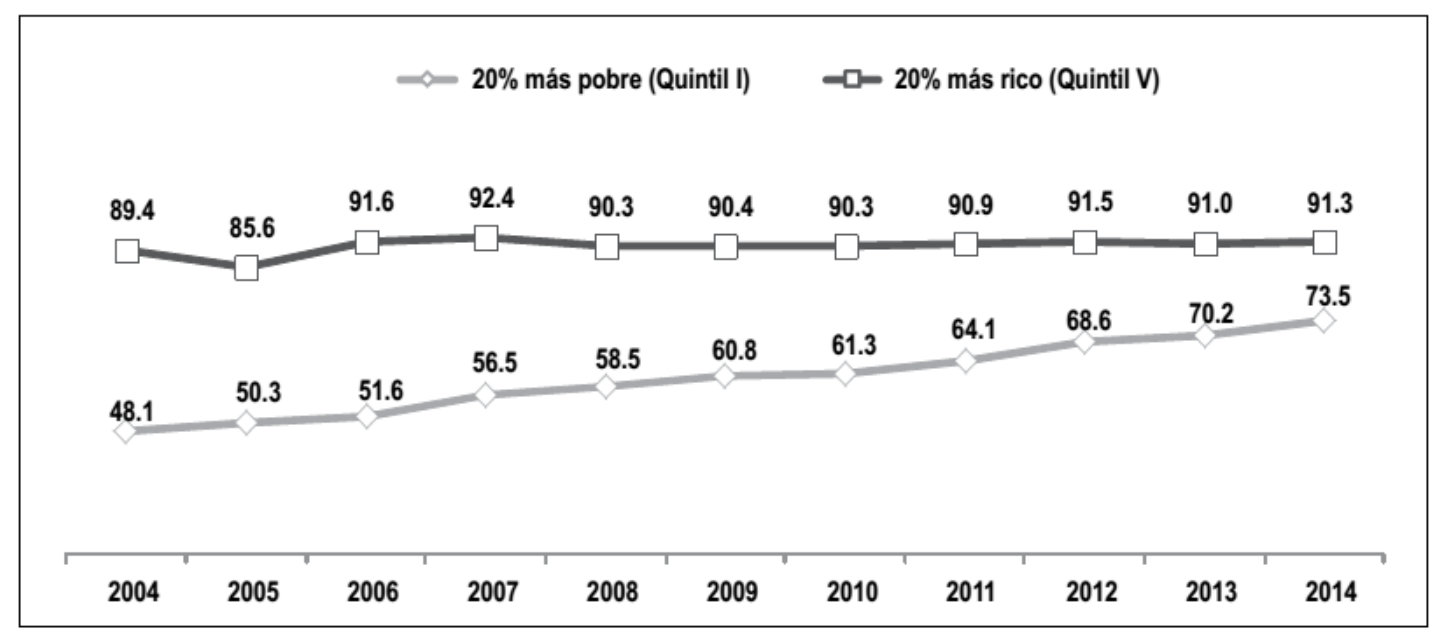

Figura 4. Perú: tasa de matrícula de educación secundaria de la población de 12 a 16 años de edad, según condición socioeconómica, 2004-2014

Fuente: Instituto Nacional de Estadística e Informática. Encuesta nacional de hogares.

Evidentemente, Perú camina hacia una inclusión de acceso, como bien lo define la Ley de Educación que les rige. Es importante los logros alcanzados en el nivel primario y es un desafío en los otros niveles. En esta síntesis no se ha incluido el detalle de la información sobre la afectación que tienen estos datos en relación a la variable sexo y zonas, pues en todos los niveles se observa que ser niña es un factor de exclusión, así como el pertenecer a una zona rural.

Nuestras percepciones sobre el Sistema Educativo en Perú no son lejanas, a la que observamos en Europa en cuanto a los objetivos que alcanza, y las prácticas excluyentes que lo caracterizan, sin duda queda mucho por mejorar a ambos lados del Atlántico. Y es singularmente llamativo que se repite, como creemos que, en todo el mundo, un modelo de Sistema Educativo Excluyente, como lo dice Echeita y Sandoval (2002), mediante mecanismos que a veces son "explícitos" (como la segregación de alumnos en colegios de mayor o menor nivel social y académico) y otras "implícitos u ocultos" (como las prácticas de selección mediante pruebas de acceso, la sobrevaloración de las capacidades de tipo intelectual, el currículum rígido, etc.).

La UNESCO (1996) señala como responsable del fracaso escolar a los diferentes tipos de violencia estructural y violencia directa que se observar en el sistema educativo cada vez más excluyente. Ángeles Parrilla (2002) hace una síntesis a lo largo de la historia de cómo ha actuado el sistema educativo en la atención de los principales colectivos discriminados (tabla 1). 
Tabla 1. Evolución de la atención del sistema educativo a los colectivos excluidos

\begin{tabular}{|c|c|c|c|c|}
\hline $\begin{array}{c}\text { PERÍODOS/COLECTIVOS AFECTADOS POR } \\
\text { FACTORES DE EXCLUSIÓN }\end{array}$ & Clase Social & Grupo CUltural & GÉNERO & DISCAPACIDAD \\
\hline Exclusión: negación del derecho a la educación. & No escolarización. & No escolarización. & No escolarización. & Infanticidio/internamiento. \\
\hline $\begin{array}{l}\text { Segregación: Reconocimiento del derecho a la } \\
\text { educación diferenciada. }\end{array}$ & $\begin{array}{l}\text { Escuelas Graduadas. En } \\
\text { España llamadas } \\
\text { "Escuelas Populares" } \\
\text { dirigidas a los sectores } \\
\text { trabajadores. } \\
\end{array}$ & $\begin{array}{l}\text { "Escuelas puentes", } \\
\text { hasta los años } 80 \\
\text { existían como } \\
\text { escuelas destinadas } \\
\text { a la cultura gitana. }\end{array}$ & $\begin{array}{l}\text { Escuelas separadas: } \\
\text { Niñas. }\end{array}$ & $\begin{array}{l}\text { Escuelas Especiales: destinados a } \\
\text { la atención de niños/as con } \\
\text { discapacidades, organizados } \\
\text { según los déficits. }\end{array}$ \\
\hline $\begin{array}{l}\text { Reformas integradoras: Se incorporan los grupos } \\
\text { excluidos a la escuela ordinaria, sin que esta se } \\
\text { modifique con lo cual supone un proceso de } \\
\text { Asimilación a la cultura dominante (en la medida } \\
\text { que fuera posible asimilarse, pues sino se excluye } \\
\text { nuevamente) y en otros casos supone sólo un } \\
\text { cambio de espacios físicos (Booth y Ainscow, } \\
\text { 1998). }\end{array}$ & $\begin{array}{l}\text { Comprehensividad } \\
\text { (entre los años 50-60). }\end{array}$ & $\begin{array}{l}\text { E. Compensatoria. } \\
\text { E. Multicultural (a } \\
\text { partir de los años } \\
\text { 80). }\end{array}$ & $\begin{array}{l}\text { Coeducación (a } \\
\text { partir de los años } \\
70) .\end{array}$ & $\begin{array}{l}\text { Integración Escolar (en España a } \\
\text { partir de los años 80). }\end{array}$ \\
\hline $\begin{array}{l}\text { Reformas inclusivas: En el estadio anterior lo que } \\
\text { falla son los procesos, hace falta una real } \\
\text { transformación centrada en procesos participativos } \\
\text { que garanticen el derecho no solo a permanecer } \\
\text { sino a participar y actuar. }\end{array}$ & Ed. Inclusiva. & $\begin{array}{l}\text { Ed. Inclusiva e } \\
\text { Intercultural. }\end{array}$ & Ed. Inclusiva. & Ed. Inclusiva. \\
\hline
\end{tabular}


Por ello aunque se valora el trabajo que hacen los docentes al querer atender a cada sujeto en sus necesidades, la educación especial y la educación compensatoria, no dejan de ser modelos excluyentes que en cierto modo siguen segregando a los alumnos dentro de la clase ordinaria, situación que aún se observa en el sistema educativo de Perú, pero que recibe el nombre de educación inclusiva.

La preocupación por hacer un sistema menos excluyente está en la base de las prácticas de una educación inclusiva. Que se fundamenta en un proceso de enriquecimiento ideológico y conceptual en relación a los planteamientos integradores (Parrilla, 2002), trasladando esta discusión al contexto social sobre el reconocimiento y la participación de los grupos excluidos en la sociedad y en la desnaturalización de los mecanismos excluyentes, como lo ha sido la escuela.

La inclusión educativa es el proceso para tratar de eliminar las barreras de distintos tipos que limitan: la presencia, el aprendizaje y la participación de cualquier alumno/a en la vida y en la cultura escolar de los centros educativos, tomando en especial consideración a aquellos que se consideran que son más vulnerables (Ainscow, Booth y Dyson, 2006).

\section{Criterios para poder observar una realidad desde la perspectiva de la educación inclusiva}

Sin ánimo de definir sino solo aclarar los puntos a observar durante la investigación se propone un breve desarrollo sobre los hitos y las experiencias que han permitido ir configurando esta idea de educación inclusiva.

Entendemos que el origen de esta nueva definición se centra en la Conferencia Mundial sobre Necesidades Educativas Especiales realizada en Salamanca en 1994, en la misma se suscribe:

El principio rector de este Marco de Acción es que las escuelas deben acoger a todos
los niños, independientemente de sus condiciones físicas, intelectuales, sociales,
emocionales, lingüísticas u otras. Deben acoger a niños discapacitados y niños bien
dotados, a niños que viven en la calle y que trabajan, niños de poblaciones remotas o
nómadas, niños de minorías lingüísticas, étnicas o culturales y niños de otros grupos
o zonas desfavorecidas o marginados... Las escuelas tienen que encontrar la
manera de educar con éxito a todos los niños... (UNESCO/MEC, 1994, p. 6)

A partir de este marco de acción podemos rescatar dos aspectos que se convertirán en unos indicadores claros de a quiénes implica la educación inclusiva:

- Pues ante la impronta del "todos" está claro que esto significa que alcanza a todos los niños que estén en riesgo de exclusión, porque no tienen posibilidad de beneficiarse de la educación (Parrilla, 2002), más allá de los que presentan NEE. Por tanto, también incluye los de minorías lingüísticas, étnicas o culturales, así como los niños de familias nómadas o de escasos recursos o niños que trabajan o viven en la calle, etc.

- En una segunda parte y con el ánimo de viabilizar la acción requerida hace especial referencia a la responsabilidad de la "escuela" no habla de diferentes tipos de escuelas, sino de una sola que acoge a todos los niños. En este caso, en un símil a lo dicho por Ainscow 1999 (citado por Echeita y Sandoval, 
2002) no es cuestión de que los niños se adapten a la escuela, sino que de una vez por todas las escuelas (entendiendo así a los sistemas educativos) entren en un profundo proceso de transformación, capaz de crear "un nuevo juego" pues con seguir cambiando las reglas de un juego equivocado ya se ha visto que no ha mejorado la situación.

Para Ainscow y Booth (1998) la educación inclusiva es un proceso para aumentar la participación de los alumnos en el currículo de las comunidades escolares y en la cultura.

Por tanto, sintetizando este apartado entendemos que los implicados y al mismo tiempo beneficiados del movimiento de la escuela inclusiva (Echeita y Sandoval, 2002) son todos los niños (sin tener en cuenta las características que lo diferencian), así como la escuela y la comunidad que le rodea.

Echeita y Sandoval (2002) y Parrilla (2002), enlistan un importante número de denominadores comunes recogidos de algunos ejemplos, que a nivel internacional, han evidenciado en sus prácticas la posibilidad de movilizarse hacía la idea de educación inclusiva (Bernal y Gil, 1999; Flecha, 1997; Slavin, 1996; Skrtic, 1999; Vargas y Flecha, 2000, etc.). Se podrían citar los siguientes:

- Es un proceso, que implica un cambio profundo, en el sistema educativo (como hemos dicho antes no cambiar más las reglas de juego, sino que es necesario cambiar el juego), iniciando el mismo desde una concepción sistémica que implique a todo el entorno social y haga que entre ambos fluya una sinergia transformadora (Echeita et al., 2014).

- Abrir las puertas de la escuela, para unificar esfuerzos de padres, docentes, administradores y agentes locales. Implicándoles en las decisiones transformadoras del entorno escolar y social. Esta implicación también en la atención y ampliación del aula como espacio de aprendizaje.

- Promoción de estrategias de liderazgo colaborativo y participativo. Favoreciendo una reestructuración de los servicios de educación especial y fomentando la colaboración y el apoyo entre los docentes y entre las familias y los agentes externos, que mejore, empodere y enriquezca la labor de enseñar.

- Desmontar las prácticas de aulas tradicionales y muchas veces prescriptas por las editoriales, incorporando la innovación en el aula desde la perspectiva del diálogo y la participación, a través de técnicas de aprendizaje cooperativo, enriquecimiento y aceleración de contenidos especialmente en la lectoescritura.

- Por último, tener siempre presente la “máxima irrenunciable” de "querer para todos los niños y jóvenes, especialmente para aquellos que están en peor situación, lo que cualquiera de nosotros quisiéramos para sus hijos" (Echeita y Sandoval, 2002, p. 42). Por lo tanto, nunca rebajar el currículum, suponiendo erróneamente que sus limitaciones físicas, culturales, sociales le van a impedir alcanzarlos, pues en tal caso estaríamos dándoles menos oportunidades y creando nuevamente circuitos diferenciados (Filmus, 2000; Filmus y Braslavsky, 1987; Sendon, 2005; Tiramonti, 2004), haciendo dentro de la escuela grupos segregados. 
A modo de síntesis, en el último libro de Booth y Ainscow (2015) denominado Guía para la educación inclusiva: desarrollando el aprendizaje y la participación en los centros escolares, aparece un breve listado (ver tabla 2) que establece unos puntos claros e importantes que nos ayudan a clarificar y sintetizar esa mirada inclusiva dentro de un centro escolar.

Tabla 2. Características de centros inclusivos

\section{LA INCLUSIÓN EN EDUCACIÓN SIGNIFICA}

Poner los valores inclusivos en acción.

Mirar cada vida y cada muerte con igual valor.

Apoyar el sentimiento de pertenencia en todos.

Incrementar la participación de los estudiantes en las actividades de enseñanza y aprendizaje, así como las relaciones con las comunidades locales.

Reducir la exclusión, la discriminación y las barreras para el aprendizaje y la participación.

Reestructurar las culturas, las políticas y las prácticas para responder a la diversidad de alumnos que aprenden de modo que se valore a todos igualmente.

Vincular la educación a realidades locales y globales.

Aprender de cómo se han reducido las barreras para el aprendizaje y la participación con algunos estudiantes para que este conocimiento beneficie a todos los estudiantes.

Mirar las diferencias entre estudiantes y entre adultos como recursos para el aprendizaje.

Reconocer el derecho de los estudiantes a una educación de calidad en su localidad.

Mejorar los centros escolares tanto para el personal, los padres y tutores, como para los estudiantes.

Enfatizar el proceso de desarrollo y mejora de las comunidades escolares y sus valores, tanto como sus logros.

Fomentar relaciones mutuamente enriquecedoras entre los centros escolares y las comunidades del entorno.

Reconocer que la inclusión en la educación es un aspecto de la inclusión en la sociedad.

Fuente: Elaborado a partir de Booth y Ainscow (2015).

A partir de estos criterios hemos realizado esta investigación de contraste entre lo observado y el marco teórico presentado, pero que entendemos como flexible y en construcción. A continuación, presentamos cómo se ha planteado la investigación y los resultados obtenidos.

\section{Método}

Se ha optado por la utilización de una metodología cualitativa entendida como una actividad sistemática orientada a la comprensión en profundidad de fenómenos educativos y sociales (Sandín, 2003). Utilizamos un enfoque interpretativo, puesto que lo consideramos más adecuado desde una doble perspectiva: permite atribuir un significado al objeto estudiado y descubrir el sentido que tiene para aquellos que lo han experimentado (Moliner y St-Vincent, 2014).

\section{Participantes}

Primero se realizó un grupo de discusión con un grupo de docentes y padres pertenecientes a un centro de educación primaria, participaron alrededor de 15 personas. En el análisis de contenido se identifican como D: a los docentes de Educación Primaria y como $\mathrm{P}$ a los padres. 
Por otro lado, también se recogió información sobre educación inclusiva a través de un grupo de alumnas de educación infantil de una universidad pública, quiénes durante una clase analizaron el tema de referencia, participaron alrededor de 40 personas. En el análisis de contenido se identifican como A: a los alumnos de magisterio que han participado de esta investigación.

También se realizaron entrevistas abiertas a profesionales del ámbito de la educación. Participaron 2 profesores universitarios. En el análisis de contenido se identifican como PU: profesores universitarios.

\section{Instrumentos}

Para los grupos de discusión se utilizaron dos tipos de instrumentos para recoger los datos: por un lado, una grabadora de voz y por otro lado un documento escrito por los propios participantes siguiendo las preguntas que guiaban la discusión. Estas preguntas eran de carácter abierto.

En las entrevistas en profundidad realizada a los profesores universitarios se utilizó una grabadora de voz y un guion con preguntas abiertas. Las preguntas guías para los grupos de discusión como para el guion de la entrevista han sido las siguientes: ¿Cómo se entiende la educación inclusiva en Perú? ¿En la práctica cómo se concreta en las aulas?, ¿Cuáles son las barreras que se identifican para su aplicación? y ¿Qué se puede hacer para mejorar la escuela de cara a la educación inclusiva?

Simultáneamente la investigadora llevaba un diario de campo donde se registró cada una de las situaciones previstas para la recogida de información y las percepciones del contexto educativo de Lima durante las visitas a los centros educativos.

\section{Procedimiento}

Se establecieron las categorías de observación y estudio. Luego se concretaron visitas a centros educativos y se realizó una observación (no participante) y posteriormente se realizó un contraste de teoría y observaciones recogidas en el diario de campo. Se platearon hipótesis explicativas sobre dichas observaciones.

A continuación, se contactó con posibles grupos de informantes de diferentes niveles educativos y con diferentes implicaciones dentro del sistema educativo, buscando abarcar a Alumnos, Padres y Docentes.

Se realizaron los grupos de discusión (2) y las entrevistas (2) siguiendo los guiones propuestos para indagar y reflexionar sobre las preguntas guías, buscando contrastar las hipótesis explicativas previas.

\section{Análisis de datos}

El sistema de registro de la información se realizó mediante documentos escritos, en algunos casos escrito por la propia investigadora (diario de campo y transcripciones de entrevistas) y en otros casos escritos por los participantes de los grupos de discusión como los documentos resúmenes o conclusiones de cada grupo.

Para su análisis hemos realizado una categorización mediante un proceso deductivo extrayendo los criterios que nos permitirían observar el desarrollo de lo que entendemos por educación inclusiva (ver tabla 3), pero también se ha estado atento al proceso inductivo, sobre situaciones que podrían emerger de estos documentos y de las observaciones. Esta categorización es una selección de factores y elementos a tener en 
cuenta, pero somos conscientes que se podría diversificar aún más por la amplitud del constructo y su práctica.

Se ha utilizado el Atlas.ti, para la reducción y organización de datos.

Tabla 3. Preguntas de investigación y categorías de análisis

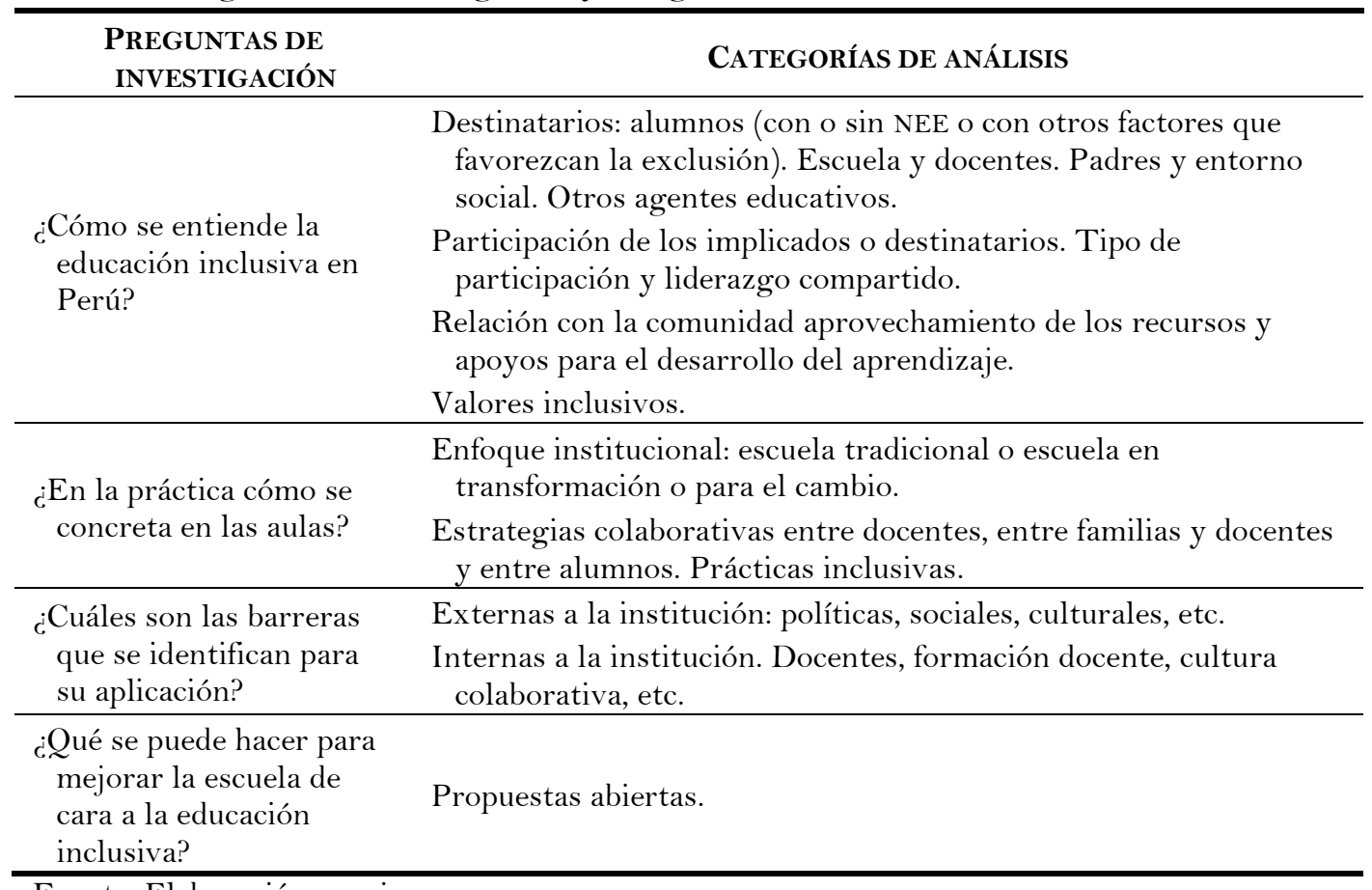

Fuente: Elaboración propia.

\section{Estrategias de evidencias científicas}

Para evidenciar las estrategias que se han seguido para consolidar el criterio de Valor de Verdad propuesto por Guba y Lincoln (1981) y dar rigor científico a esta investigación hemos utilizado:

- La triangulación, que significa conseguir varias aproximaciones de una misma realidad, para asegurar que las interpretaciones son correctas (Bartolomé, 1996). Este postulado se refleja del siguiente modo: Triangulación de informantes: Por el cual han participado en la recogida de datos diferentes grupos de personas, teniendo en cuenta: la implicación en relación a la escuela (docentes, padres, alumnos de magisterio, profesores de universidad).

- Triangulación de técnicas y fuentes: se han utilizado diferentes técnicas y recursos para recoger los datos. Además, se ha combinado la observación de los centros educativos y la lectura de los informes oficiales emitidos por el Estado Peruano.

- Observación prolongada: el proceso de observación en los centros educativos de diferentes niveles ha sido prologada, aproximadamente dos meses. Lo que ha permitido ir reconfirmando las afirmaciones que habían resultado de los diversos informantes. 
- Comentario de pares y comprobaciones con los participantes, en este criterio se han compartido los resultados y las interpretaciones con los profesores universitarios de Perú, quienes al mismo tiempo que han sido participantes durante el estudio, han podido matizar las interpretaciones y reflexiones que han surgido del total de los datos analizados.

El criterio del Valor de Verdad hace referencia a la credibilidad o validez interna de la investigación. Este criterio busca que los datos recogidos sean imagen de la realidad que se estudia evitando el sesgo o subjetividad del observador. Pero al mismo tiempo se sabe que el fin último de estos datos no es la generalización sino un punto de partida que sirva para la reflexión de los implicados en el proceso.

\section{Resultados}

A continuación, se presentan los resultados que se han recogido de los diferentes informantes y fuentes, dicha presentación hace referencia a cada una de las preguntas que han guiado la investigación, y a las categorías de análisis establecidas previamente.

\section{1. ¿Cómo se entiende la educación inclusiva en Perú?}

En este apartado se recogen datos relativos a los agentes implicados o destinatarios, así también su nivel de participación, y a los procesos y cambios institucionales en pos de la educación inclusiva.

\section{Los destinatarios y agentes implicados en la educación inclusiva}

En cuanto a los alumnos: Desde las diferentes fuentes de información se puede observar que se ha dado un paso en la integración de los niños con discapacidades en las aulas ordinarias públicas, en una situación semejante a la que describe Parrilla (2002) para este periodo. Situación que dista de lo que hemos explicado sobre educación inclusiva en los párrafos anteriores. También hay que enfatizar que entre los participantes se entiende que la educación inclusiva abarcaba sólo a los niños con discapacidades, pero no a todos los otros grupos de niños excluidos de la escuela. Una contradicción entre el reconocimiento de que la educación es un derecho para todos, pero al mismo tiempo un reconocimiento que en ese "todos" no están incluidos los niños que trabajan, los que son de escasos recursos económicos, o que tienen cualquier tipo de dificultad para acudir a la escuela.

Se rescata este comentario de una alumna de magisterio: "Bueno en nuestra realidad vemos que muchos niños no estudian por estar trabajando o por el simple hecho de que sus posibilidades económicas no lo permiten."(A2). Otras voces aseguran: "aquí en el Perú se ve mucho de eso...” (otra alumna de magisterio A5); “y estas diferencias se enfatizan aún más en las zonas urbanas y rurales..." (A3)

Estas últimas afirmaciones coinciden con las estadísticas proporcionadas por el INIE (2015).

En cuanto a los docentes: Se observa la tarea del docente desde una acción asistencial y solidaria, en tanto acepta (como quién hace un favor) el incluir un niño con discapacidad en el aula. En todas las fuentes (Alumnos de Magisterio, Docentes, Padres y Profesores Universitarios) se reconoce al docente como un sujeto mediador del cumplimiento de la ley y la adaptación del aula y del curriculum de modo individual para el alumno con NEE. 
Una reflexión positiva sobre la responsabilidad del docente ante la educación inclusiva, elaborada por unos alumnos de magisterio relata: "para una buena educación es necesario buenos docentes que se preocupen en innovar y realizar diferentes estrategias para el buen aprendizaje del alumno, no solo para aquel que tenga las posibilidades económicas..." (A1)

De esta frase rescatamos, lo dicho en el apartado anterior, se ve la inclusión como la adaptación e innovación de estrategias, no se mencionan si se tienen en cuenta estrategias cooperativas y de interacción en el aula. También se considera oportuno poner en contexto lo dicho con respecto a "no solo para aquel que tenga las posibilidades económicas” esto hace referencia al sistema educativo de Perú en el que existen simultáneamente circuitos diferenciados para ricos y pobres, y sin duda, generan una violencia estructural, que se traduce, como ya se ha dicho, en el alto índice de fracaso escolar, especialmente en el nivel de secundaria.

Los docentes además reconocen su falta de formación para generar los cambios hacia la educación inclusiva tal como se expone a continuación: "Creo que si estos grupos de cambio se dan en España aquí también se puede hacer aunque nos falta más formación” (D5); "los docentes debemos ser positivos, creadores, pacientes, motivadores, etc. para encaminar al niño no solo exigiendo sino brindando posibilidades” (D3).

También vemos como el docente que reflexiona sobre estos temas se empodera para iniciar el cambio hacia la educación inclusiva, por ello, algunos de los docentes participantes en los grupos de discusión afirman: "El cambio empieza por nosotros" (D1)

En cuanto a la comunidad escolar (incluido otros agentes sociales que rodean a la escuela), se puede decir que, en los casos observados, en las escuelas y los grupos de discusión, este tema resultaba de gran rareza, por lo que para ellos es impensable que otros agentes puedan intervenir y participar en la actividad escolar. Esta participación se reduce sólo a actividades festivas y solidarias, pero no incluye una participación ni en las prácticas docentes en el aula, ni en el curriculum del centro escolar.

En el diario de campo de la investigadora se recoge con frecuencia que en las infraestructuras de los centros educativos existe una puerta simbólica y grande a la entrada, pero que permanece cerrada y que para acceder se debe pasar por una puerta pequeña anexa o dentro de la más grande y casi siempre a cargo de la vigilancia de un portero. Situación que se justifica por los problemas de seguridad que azota al país, pero por otro lado hace referencia a la desconexión de la escuela con su entorno ya que teme a sus propios vecinos.

Destacamos un ejemplo de una alumna de magisterio: "me ha sorprendido que diga que se debe invitar a los padres de familia y a la comunidad a participar a la escuela" (A1)

También comentar que el ingreso a cada centro además de estar mediado por el portero, depende de la autorización del director. La figura del director es claramente el representante de la autoridad; que, según las anotaciones del diario de campo de la investigadora, tanto la imagen personal y el trato con el resto del personal docente es de sumisión a dicha autoridad, con lo cual no existe la posibilidad de hablar de un liderazgo distribuido o colaborativo. Pero se entiende que el modelo de liderazgo autoritario, se imponga en los centros educativos, pues están influidos por los mismos valores que impregnan la cultura, pues si no hay un cambio cultural previo sobre estos valores, el 
entorno de la escuela podría percibir a la dirección del centro como incompetente para el cargo si su comportamiento fuera más flexible y cercano.

Como ya se ha comentado, se entiende la Educación Inclusiva como la incorporación de los niños con NEE a las escuelas ordinarias, produciéndose solo un cambio de ubicación física de estos niños. No hay lugar para observar ni rescatar la concepción procesual y sistémica que implica el gradual desarrollo de la educación inclusiva, como tampoco, según ya se ha comentado, la participación abierta a las familias y a la comunidad, ni el liderazgo compartido. Por ello se puede suponer que la principal barrera a sortear para la implementación de la educación inclusiva es el cambio de ciertos conceptos culturales que impregnan a la actual escuela de un estilo claramente tradicional y elitista.

\section{2. ¿En la práctica cómo se concreta la educación inclusiva en las aulas?}

En este apartado se manifiestan las percepciones de los diferentes participantes sobre cómo ellos ven que ésta propuesta se concreta en el aula a través de sus estrategias evidenciando el enfoque institucional que subyace a dichas prácticas.

\section{La realidad de la implementación de la ley que avala la educación inclusiva}

Se reconoce que la definición que hace actualmente la ley de educación (de Perú 28.440, en su reforma de 2012) sobre inclusión dista de lo que es la formación del docente para poder implementarla, de la infraestructura necesaria y de lo que realmente se ha llegado a poner en práctica. Como se ha comentado anteriormente sólo se ha integrado a algunos alumnos con NEE en los centros ordinarios. Los docentes dicen: "Existe una sobrecarga de alumnado que impide que el docente cumpla con su rol" (D2); "El proyecto de inclusión está mal implementado" (D4); "Necesitamos formación en estrategias de aula" (D3). Estas frases reflejan en cierto modo la distancia entre la ley que les regula y las necesidades que reclaman los docentes para poder ser protagonistas de estas implementaciones.

Hay que destacar que la carrera profesional del docente en Perú está muy mediatizada por el dinero que se pueda invertir en hacer cursos de formación, pues no solo se trata de juntar méritos académicos, sino que muchos o casi todos están vinculados a un costo que en algunos casos carecen de calidad. En estos últimos años se ha creado una entidad para tratar de regular la calidad de la formación SINEACE (Sistema Nacional de Evaluación, Acreditación y Certificación de la Calidad Educativa). Muchos de estos centros de formación docente son de carácter privado y aún no están acreditados.

También entre los análisis ha emergido una singularidad del vocabulario propio de Perú y que ejemplifica claramente la concepción segregacionista que aún se respira en el sistema educativo. Un ejemplo, durante el recorrido por una escuela ordinaria de educación infantil, la investigadora relata en el diario: cuando entraba en un aula la persona responsable de acompañarnos solía presentarnos al grupo y señalar con orgullo y en voz alta: "acá tenemos un alumno inclusivo" y nos lo presentaba (diario de campo pág. 16). Esto mismo se observa en el leguaje de los docentes que participan de la investigación y de los alumnos de magisterio. Nuevamente esos alumnos que ahora están en un centro ordinario reciben una etiqueta "alumno inclusivo".

Estrategias en el aula 
Las estrategias de aula que con más frecuencia se ha recogido es la adaptación curricular para los niños con NEE. Esta adaptación implica habitualmente una disminución del currículum.

Mayoritariamente se sigue un modelo tradicional mediado hoy en día por los libros escolares de las diferentes asignaturas. Donde los niños interaccionan muy poco entre ellos para resolver situaciones problemáticas o proyectos de investigación: "Su principal interacción o acción motriz está relacionada con la tarea que propone el libro" (Diario de campo p. 24).

\section{3. ¿Cuáles son las barreras que se identifican para la aplicación de la educación inclusiva?}

Ante esta cuestión, las respuestas las podríamos agrupar en dos aspectos:

A nivel externo de la escuela: Se considera que la sociedad, y especialmente los padres de otros alumnos, no están concienciados de la necesidad y el derecho de integrar a todas las personas independientemente de sus diferencias. La visión que existe, por parte de la sociedad, de una escuela que integra los distintos tipos de NEE, es que el nivel académico de dicha institución es muy bajo tanto que si es posible los padres no lo elegirían para la educación de sus hijos. No se perciben los valores de solidaridad y cooperación como bienes que se pueden adquirir en la escuela, pues el perfil que proponen las escuelas, especialmente las privadas, es el de un éxito social basado únicamente en el mérito académico. Algunas de las respuestas que lo ejemplifican dicen: "Todos (docentes, padres y alumnos) deben sentirse comprometidos a generar cambios hacia la flexibilidad" (D1); "No se ha previsto una campaña de sensibilización a la sociedad civil" (P2); "Otra barrera son los prejuicios de la sociedad" (A1).

A nivel interno de la escuela: las barreras detectadas se centran en dos ámbitos, por un lado (y con más frecuencia de aparición) la falta de capacitación de los docentes para innovar y aplicar estrategias que favorezcan el trabajo cooperativo en el aula. Y por otro lado, otra cuestión que se identifica como barrera, es el elevado número de alumnos por clase o docente y que en cierto modo sirve de justificación para seguir utilizando las metodologías tradicionales y las adaptaciones curriculares como medida de atención a la diversidad. Algunos comentarios de docentes y padres rescatan que las principales barreras son: "Falta de preparación de los docentes" (D4); "Carga docente inadecuada" (D2); "la carga de alumnado para el docente" (P1).

\section{4. ¿Qué se puede hacer para mejorar la escuela de cara a la educación inclusiva?}

Las propuestas que se han realizado, desde los grupos de discusión y las entrevistas, son efectivamente respuestas a las barreras que se han detectado en el apartado anterior. Por ello entre las propuestas citamos: "Una posibilidad de futuro sería capacitar y sensibilizar a los docentes en su desarrollo profesional, para que aplique estrategias con sus educandos" (D2); "Generar talleres educativos con alumnos, padres y docentes" (P1); "Reformular la malla curricular en la formación pedagógica incidiendo en la educación inclusiva e intercultural" (PU2). Por tanto, se puede sintetizar la propuesta de acción para desarrollar la educación inclusiva en formar y sensibilizar a los docentes y a la sociedad en general. 


\section{Conclusiones}

Tal como se ha presentado en los resultados, vemos que la percepción que tienen los participantes acerca la educación inclusiva es muy semejante al estadio que Parrilla (2002) describe como de "integración", en tanto, se ha dado un paso importante en el reconocimiento de los derechos de las personas con NEE, a estar en una clase de un centro ordinario. Pero esta incorporación sólo ha significado un cambio de ubicación física, incluso se observa una etiquetación a dichos niños como "alumnos inclusivos", situación que en el Sistema Educativo de México se repite y que describe Romero y García (2013) cuando dice que los niños que se integran en las aulas ordinarias se etiquetan como "niños de apoyo". En ambos casos la etiquetación es un síntoma de segregación dentro del aula. Lo que en palabras de Echeita y Sandoval (2002) se identifican como mecanismos implícitos u ocultos de un sistema educativo excluyente.

Entre los participantes de la investigación existe un pensamiento común: "en Perú de ningún modo se ha pensado en que la educación inclusiva es una reivindicación para el reconocimiento de los otros grupos excluidos de la escuela y de la sociedad" (P2). Sin embargo, la tasa neta sobre el acceso de los alumnos de escasos recursos económicos en los últimos años se ha elevado (INIE, 2015) este pensamiento contradictorio entre la percepción de los actores educativos y la realidad de los números que expone el Estado Peruano, podría ser un tema a profundizar en futuras investigaciones.

Tampoco se piensa que la educación inclusiva deba asentarse en la participación, especialmente de los padres, en la vida y las decisiones de la escuela. La escuela se percibe como una entidad exclusiva, y herméticamente cerrada a las necesidades de la sociedad, solo responde a una demanda meritocrática del mercado de capitales, por lo cual los menos hábiles y útiles para la sociedad se deben quedar fuera del sistema pues estos no alcanzan a adaptarse a sus exigencias. Para nosotros que entendemos que la escuela inclusiva percibe la demanda de modo contrario, respondiendo a las necesidades de la sociedad, que reclama cohesión y colaboración, para dar respuesta a los que más necesitan tratando de cumplir el mandato de la equidad.

Por todo esto vemos que hablar de educación inclusiva en Perú dista mucho de lo que desde la teoría queremos reconocer al respeto.

Por ello, no solo la escuela en Perú, sino que, en muchas partes del mundo, necesita iniciar un proceso de cambio profundo que transforme los valores que sostienen una cultura cada vez más excluyente a nivel educativo y a nivel social. Citando la propuesta de la UNESCO (2008):

Debemos de considerar a la educación como un factor de cohesión social procurando transformar la diversidad en un factor positivo de entendimiento mutuo entre los individuos y grupos humanos y al mismo tiempo evitar ser ella misma un factor de exclusión social. (p. 5)

El principal reto se vive en las aulas, pues una vez más se ve que no basta con la promulgación de leyes con palabras ejemplares para que se produzcan los cambios tan esperados, sino que son los maestros los principales artífices del proceso de cambio y por ello son los primeros que se deben involucrar mediante la formación y el compromiso que esto exige, y que hoy por hoy sigue siendo una tarea pendiente del gobierno de Perú, pues la implementación de la ley no puede estar condicionada a la disponibilidad económica de los mismos docentes. 
Son los mismos docentes los que manifiestan la necesidad de formación y a nivel estructural la disminución de alumnos por aulas. También estos procesos deben ser acompañados por procesos de sensibilización social ante la realidad de la exclusión, entendemos importante romper con la naturalización de la exclusión y la insensibilidad que rodea a las personas que sin saberlo actúan, sin mala intención, como ejecutores de la condena social clasista. Una vez más estos rasgos del sistema educativo peruano hace evidente estos mecanismos implícitos u ocultos (Echeita y Sandoval, 2002) que lo definen como excluyente. Pero que más allá de ser una crítica es el primer paso para el cambio, basado en el conocimiento de una realidad, sus barreras y sus potencialidades. Sobre esto último, hay que destacar la fuerza y ganas que los docentes muestran en su tarea diaria y el reclamo y deseo de aprender más para hacer mejor su trabajo son los pilares para impulsar estos cambios (Escobedo y Sales, 2012).

Todo esto nos posiciona junto a las palabras de Leiva y Gómez (2015) ante un complejo reto, pues en la actualidad defendemos la idea de que la educación inclusiva se encuentra entre el deseo de ser una propuesta educativa crítica, y la realidad de encontrarse en una encrucijada de caminos que van desde la segregación encubierta del alumnado con necesidades educativas especiales a la falta de profesionales especializados, así como la escasa formación en educación inclusiva de todo el profesorado y la comunidad educativa. Lo que se traduce en la necesidad de una gran dosis de optimismo que debe ir acompañando un desarrollo progresivo en todos los niveles del sistema educativo.

Es hora de repensar lo que Calderón (2016) llama la escuela de la esperanza. Y es por ello que una investigación como esta tiene sentido, pues la reflexión y la construcción dialógica y crítica de la realidad entre los actores de la escuela peruana es el inicio de un camino que no puede volver atrás y que debe mirar de modo proactivo la necesidad de anticiparse a una realidad que reclama una justicia social inminente. La escuela de los estándares y el acatamiento no es la única posible.

\section{Referencias}

Ainscow, M. (2004). El desarrollo de sistemas educativos inclusivos: ¿¿uáles son las palancas de cambio? Journal of Educational Change, 5(4), 1-20. doi:10.18172/con.585

Ainscow, M., Booth, T. y Dyson, A. (2006). Improving schools, developing inclusion Londres: Routledge.

Bernal, J. y Gil, M. (1999). Escuelas aceleradas. Un sueño que se hace realidad. Cuadernos de Pedagogía, 285, 33-38.

Booth, T. y Ainscow, M. (1998). From them to us. An international study of inclusion on education. Londres: Routledge.

Booth, T. y Ainscow, M. (2015). Guía para la educación inclusiva: desarrollando el aprendizaje y la participación en los centros escolares. Madrid: OEI.

Calderón, I. (2016). Liberarse de la escuela. Historia de vida de Elena. Málaga: Ruima.

Echeita G. y Sandoval, M. (2002). Educación inclusiva o educación sin exclusiones. Revista de Educación, 327, 31-48.

Echeita, G., Muñoz, Y., Sandoval, M. y Simón, C. (2014). Reflexionando en voz alta sobre el sentido y algunos saberes proporcionados por la investigación en el ámbito de la educación inclusiva. Revista Latinoamericana de Inclusión Educativa, 8(2), 25-48. 
Escobedo, P. y Sales, A. (2012). El cambio que viene del aula: estrategias y recursos para la educación inclusiva. Quaderns Digitals, 71, 1-24.

Filmus, D. (2000). Cada vez más necesaria, cada vez más insuficiente: escuela media y mercado de trabajo en épocas de globalización. Buenos Aires: Santillana

Filmus, D. y Braslavsky, C. (1987). Último año del colegio secundario y discriminación educativa. Buenos Aires: Miño y Dávila.

Fecha, R. (1997). Compartiendo palabras: el aprendizaje de las personas adultas a través del diálogo. Barcelona. Paidós.

Guba, E. G. y Lincoln, Y. S. (1981). Effective evaluation. San Francisco, CA: Jossey Bass.

INIE (2015), Perú: Indicadores de Educación por departamentos, 2004-2014. Consultado el $15 / 12 / 15$ https://www.inei.gob.pe/media/MenuRecursivo/publicaciones_digitales/Est/Lib1293/i ndex.html

Leiva, J. y Gómez, M. (2015). La educación inclusiva como constructo pedagógico en el alumnado universitario de educación primaria. Revista de Educación Inclusiva, 8(2), 185-200.

Moliner, O. y St-Vincent, L. A. (2014). Dilemas éticos de los investigadores que acompañan procesos de investigación-acción en el marco de la escuela intercultural inclusiva. Revista Latinoamericana de Inclusión Educativa, 8(2), 49-68.

Padin, G. (2013). La educación especial en Argentina. Desafíos de la educación inclusiva. Revista Latinoamericana de Inclusión Educativa, 7(2), 47-61.

Parrilla, A. (2002). Acerca del origen y sentido de la educación inclusiva. Revista de Educación, $327,11-29$.

Ramos, L. (2013). Educación especial y educación inclusiva en Chile: ¿en punto de estancamiento? Revista Latinoamericana de Inclusión Educativa, 7(2), 37-46.

Romero, S. y García I. (2013). Educación especial en México. Desafíos de la educación inclusiva. Revista Latinoamericana de Inclusión Educativa, 7(2), 77-91.

Sandín, M. (2003). Investigación cualitativa en educación. Fundamentos y tradiciones. Madrid: McGraw Hill.

Sendon, M. A. (2005). Los egresados de la escuela media en una sociedad mutada. Revista Mexicana de Investigación Educativa, 10(24), 191-219.

Skrtic, T. (1999). Discapacidad y democracia. Voz, colaboración e inclusión en la enseñanza y la sociedad. En Grupo de Investigación HUM 181 (Coord.), Actas del seminario internacional sobre políticas contemporáneas de atención a la diversidad: repensar la educación (especial) en el tercer milenio (pp. 29-86). Salamanca: SID.

Tiramonti, G. (2004). La fragmentación educativa y los cambios en los factores de estratificación. La trama de la desigualdad educativa. En G. Tiramonti (Comp.), Mutaciones recientes en la escuela media (pp. 15-45). Buenos Aires: FLACSO.

UNESCO. (2008). La educación inclusiva. El camino hacia el futuro. Conclusiones finales de la 48 Conferencia Internacional. Consultado el $07 / 10 / 16$ en: http://www.ibe.unesco.org/fileadmin/user_upload/Policy_Dialogue/48th_ICE/CONFI NTED_48_Inf_2_Spanish.pdf

UNESCO. (1996). La educación encierra un tesoro. Informe de la comisión internacional sobre la educación para el siglo XXI. Madrid: Santillana. 
UNESCO/MEC. (1994). Informe final. Conferencia mundial sobre necesidades educativas especiales: acceso y calidad. Madrid: Ministerio de Educación y Cultura.

Vargas, M. y Flecha, R. (2000). El aprendizaje dialógico como experto en resolución de conflictos. Contextos Educativos, 3, 81-88.

\section{Breve CV de la autora}

\section{Paola Ruiz-Bernardo}

Doctora en Educación, Licenciada en Pedagogía, y profesora del Departamento de Educación de la Universitat Jaume I de Castellón. Ha realizado trabajos de investigación sobre diversidad cultural y las actitudes hacia ella como la sensibilidad intercultural y tiene algunas publicaciones y comunicaciones sobre esta temática. Investigadora del Grupo MEiCRI (Mejora Educativa y Ciudadanía Crítica). Sus líneas de investigación actuales son la educación intercultural e inclusiva y las variables contextuales de las escuelas, así como la innovación en las prácticas de aula. Universitat Jaume I de Castellón, Facultad de Ciencias Humanas y Sociales. Departamento de Educación. Área Didáctica y Organización Escolar. Email: ruizma@uji.es 